УДК: 53.091

\title{
ОСОБЕННОСТИ СТРУКТУРИРОВАНИЯ ПРИ МАССОВОЙ КРИСТАЛЛИЗАЦИИ РАСПЛАВОВ АІ И Рb В УСЛОВИЯХ ВЫСОКОИНТЕНСИВНОЙ ПЛАСТИЧЕСКОЙ ДЕФОРМАЦИИ ПРИ ЦЕНТРИФУГИРОВАНИИ
}

\author{
(2018 Ю. И. Тарасов, В. В. Крячко, В. И. Новиков \\ ООО «СВЕТОЗАР», Солянка, 1/2, 109028 Москва, Россия \\ e-mail:vinovikov@redstaratom.ru \\ Поступила в редакцию 14.02.2018
}

\begin{abstract}
Аннотация. Представлены экспериментальные результаты по кристаллизации $\mathrm{Al}$ и $\mathrm{Pb}$ в условиях высокоинтенсивной пластической деформации $\left[\varepsilon^{\prime}=\left(10^{2}-10^{4}\right)\right.$ сек $\left.^{-1}\right]$ на границе раздела «твердое-жидкое» в аппарате центробежного литья нового типа при скоростях вращения ротора до 2000 об/мин. В объёме закристаллизованных металлов $\mathrm{Al}$ и $\mathrm{Pb}$ методом атомной силовой микроскопии обнаружены вакансионные кластерные трубки со средними диаметрами $\mathrm{Al}-39$ нм, $\mathrm{Pb}-25$ нм. Обсуждается физическая модель образования в металлах новой субструктуры в виде вакансионных кластерных трубок, полученных при высокоинтенсивной пластической деформации.
\end{abstract}

Ключевые слова: интенсивная пластическая деформация, динамическая рекристаллизация, неравновесные вакансии, вакансионные кластерные трубки, твердофазная перекристаллизация.

DOI: https://doi.org/10.17308/kcmf.2018.20/484

\section{ВВЕДЕНИЕ}

В последние годы физики и механики, занимающиеся задачей повышения конструкционных свойств металлических материалов, особое внимание уделяют возможности ее решения посредством больших термомеханических режимов пластической деформации [1]. Однако на сегодняшний день механизмы деформации металлических наноструктурированных материалов и происходящие в них изменения структуры и их свойств как при нагреве, так и при больших пластических деформациях малоизучены. Нанодисперсные металлические среды (НMC) со средним размером зерна менее 0.1 мкм представляют собой в некотором отношении модельную систему для изучения структурных особенностей в объектах с сильно развитой сеткой границ (границы составляют до 5 \% объема поликристалла) при термомеханической интенсивной пластической деформации (ИПД). Характерное отличие НМС от поликристаллических систем обычной (микронной) дисперсности состоит в том, что в них благодаря размерным эффектам [2-5] могут возникать новые структурные элементы при воздействии температуры и интенсивной пластической деформации.

Проведенные исследования группой ученых (Свиридой С. В., Новиковым В. И. и др.) процессов рекристаллизации в НМС с характерным размером зерен (частиц порошка) порядка 10-100 нм методом позитронно-аннигиляционного анализа установили, что рекристаллизация сопровождается кинетическим эффектом повышения концентрации вакансий до значений $>10^{-4}$, соответствующих предплавильным. В НМС кратковременная $(t \sim 5$ мин) «инжекция» избыточных вакансий обуславливает активацию таких диффузионных процессов, как спекание, низкотемпературное растворообразование, жидкоподобная коалесценция частиц, рост крупных монокристальных образований, вакансионное кластерообразование [2-5]. В то же время, как показали специальные исследования, в сильнодеформированных НМС отсутствует дислокационная структура $[6,7]$, что находится в хорошем согласии с теоретическими оценками кри- 
тического размера дислокационной петли (0.5-1.5 мкм) [8]. Таким образом, в подобных системах отсутствуют решеточные дислокации деформации, являющиеся основным стимулом первичной рекристаллизации [1].

В то же время подобный механизм первичной рекристаллизации не давал ответа на основной вопрос, каким образом возникают и образуются новые области рекристаллизованной фазы, отделенные большеугловой границей.

Идеи, выдвинутые С. С. Гореликом и Г. Г. Глейтором $[9,10]$ в середине 80 годов, позволили по-новому рассмотреть процесс динамической рекристаллизации в сильно деформированных металлах при нагреве. Они связали процесс миграции границ с необходимостью образования избыточных (неравновесных) вакансий. Образование избыточных вакансий движущейся границей - это своеобразная «выплата» границы за возможность своего фронтального перемещения, связанная с особенностями ее строения [11]. Образование избыточных вакансий в тылу движения в этом случае не представляет собой обычного процесса диффузионного перераспределения зернограничной пустоты в объеме зерна и, по-видимому, целесообразнее говорить о «генерации» неравновесных вакансий (создание дополнительных атомных узлов решетки) кинетического происхождения. В данной ситуации рождение избыточных вакансий должно сопровождаться эффектом положительной дилатации объема, связанного с образованием дополнительных атомных узлов в решетке рекристаллизующегося объема, что экспериментально подтверждается исследованиями [2, 4]. На основе анализа процесса миграции границ при рекристаллизации НMC авторами [12] показано, что в течение короткого промежутка времени (порядка нескольких минут) уровень концентрации “динамических" вакансий в НМС может быть сравним с уровнем концентрации термодинамических вакансий при температурах, близких к температурам плавления, что находится в хорошем согласии с экспериментом [2-5].

Возможность твердофазной перекристаллизации в «заметаемом» границей объеме при зернограничном проскальзывании (ЗГП) частиц в условиях пластического сдвига приводит к образованию монокристальных «зародышей» в приграничных областях (области ЗГП) [13]. В этой связи обычное понятие о критическом зародыше не приемлемо для случаев рекристаллизации и пластической деформации. По-видимому, оправданнее говорить о «беззародышевом» механизме образования новых зерен в меру значительного пересыщения неравновесными вакансиями, приводящего к твердофазной перекристаллизации $[3,5,6,12,13]$. Одновременно на фоне высокой концентрации избыточных вакансий возникают вакансионные кластерные образования с огранкой, соответствующей типу решётки металла $[12,13]$.

Как уже упоминалось выше, основная проблема получения объёмных структурированных материалов методами термомеханической интенсивной пластической деформации металлов в промышленных масштабах ограничивается малыми размерами получаемых изделий и большой трудоемкостью их изготовления. Наибольшее использование получила технология равноканального углового прессования (РКУП), позволяющая получать объемные мелкозернистые структуры в виде прутков диаметром до 60 мм и длиной до 200 мм. Для получения более массивных структурированных материалов используется всесторонняя ковка. Таким методом ИПД, например, получают заготовки диаметром до 200 мм и длиной 300 мм из титана и его сплавов с размерами зерен 300-400 нм. Понятно, что все это не позволяет организовать крупнотоннажное производство структурированных металлов с повышенными конструкционными свойствами и, соответственно, их полномасштабное использование в создании инновационных изделий.

Основываясь на вышеизложенных физических принципах получения структурированных металлов методом термомеханической интенсивной пластической деформации, нами была поставлена задача реализовать размерный эффект динамической рекристаллизации [13] в режиме высокоинтенсивной пластической деформации (ВИПД) в металле в момент его массовой кристаллизации с целью получения новых конструкционных материалов с улучшенными свойствами в больших объемах.

\section{МЕТОДИКА ЭКСПЕРИМЕНТА}

Для решения этой задачи был создан аппарат центробежного литья новой конструкции. Были сконструированы вертикальные аппараты центробежного литья со скоростью вращения ротора до 2000 об/мин. Такие высокие скорости вращения ротора позволили создать режим высокоинтенсивной пластической деформации в твердой застывшей фазе закристаллизованного металла. Сдвиговая деформация в аппаратах такой конструкции при центробежной кристаллизации вызвана, прежде всего, большим градиентом температурного поля от периферии (относительно холодной стенки ро- 
тора) к расплавленной центральной части ротора. Разность угловых скоростей перемещения уже застывшей части металла (прилегающей к внешней поверхности стенки ротора) и центральной части, где металл еще остается в расплавленном состоянии, приводит к высокоинтенсивной деформации $\left[\varepsilon^{\prime}=\left(10^{2}-10^{4}\right)\right.$ сек $\left.^{-1}\right]$ твердой застывшей фазы закристаллизованного расплава металла. Поскольку размеры зерен закристаллизованной фазы в начальный момент составляют порядка десятков нанометров (порядка размера зародыша кристаллизации), то возникает возможность для реализации размерного эффекта динамической рекристаллизации [12, 13] «нанокристаллического» затвердевшего металла при высоких скоростях сдвиговой деформации.

Эксперименты проводились с использованием таких металлов как алюминий и свинец. Кристаллизация металлических расплавов алюминия и свинца с заданной массой и первоначальной температурой расплава производились при их объемном переохлаждении в нестационарных условиях (высокоинтенсивной пластической деформации) с заданными во времени режимами воздействия поля центробежных сил, определяемого параметрами центрифугирования, а также температурнотепловым воздействием на расплав от контактирующего с ним газового теплоносителя. Скорость охлаждения материала устанавливалась в интервале 0 до $100^{\circ} \mathrm{C} / \mathrm{c}$. Форма материала в процессе обработки представляет собой полый вращающийся вокруг своей оси цилиндр (ротор), в полость которого с заданными во времени значениями расхода и температуры вводится горячий газовый агент. Коэффициент перегрузки обеспечивался в диапазоне от 200 до 1000 g, в зависимости от требуемых характеристик интенсивности пластической деформации кристаллизующейся фазы металла. Масса материала составляла от 50 до 200 кг.

Структурные исследования выполнялись методом атомной силовой микроскопии (ACM) на зондовом микроскопе Femto Skan и Solver. P47. Динамические механические свойства изучались на приборе Q800 в атмосфере сухого воздуха при скорости нагрева $5 \mathrm{\epsilon}$ /мин в режиме консольного изгиба при заданной амплитуде 15 мк и частотой колебаний 1 гц. Испытания проводились на образцах в виде пластин размерами: длинной 35 , шириной около 4 и толщиной примерно 2.5 мм.

\section{ОБСУЖДЕНИЕ РЕЗУЛЬТАТОВ}

На (рис. 1-5) представлены результаты структурных исследований металлов $\mathrm{Al}$ и $\mathrm{Pb}$, получен- ных в условиях высокоинтенсивной пластической деформации в момент их кристаллизации. Как видно из представленных данных, в объёме закристаллизованных металлов $\mathrm{Al}$ и $\mathrm{Pb}$ обнаружены вакансионные кластерные трубки (ВКТ) со средними диаметрами для $\mathrm{Al}-39$ нм, а для $\mathrm{Pb}-25$ нм. Трубки вытянуты к центру вращения ротора (рис. 1, 3, 4). Вакансионные кластерные трубки в поперечном сечении имеют гексагональную симметрию (рис. 1,4$)$, что соответствует ГЦК решеткам $\mathrm{Al}$ и $\mathrm{Pb}$ и является дополнительным свидетельством того, что именно в закристаллизованной области фронта кристаллизации происходит формирование BКТ. При кристаллизации $\mathrm{Al}$ и $\mathrm{Pb}$ при высокоинтенсивной пластической деформации (ВИПД) порядка $\varepsilon^{\prime}=\left(10^{2}-10^{4}\right) \mathrm{c}^{-1}$ в условиях специально подобранных режимов кристаллизации металла в аппаратах высокоскоростного центробежного литья создаются условия для реализации размерного эффекта динамической (сдвиговой) рекристаллизации $[13,14,15]$.

Образующиеся при этом неравновесные вакансии конденсируются в вакансионные кластеры, которые в поле центробежных сил формируются в виде вакансионных кластерных трубок, вытянутых к центру вращения ротора (рис. 1, 3, 4). Обработка данных вакансионных трубчатых структур (рис. 1, 4) с учетом образовавшей «свободной пустоты» и времени процесса вакансионного кластерообразования дает оценочные значения концентрации не-

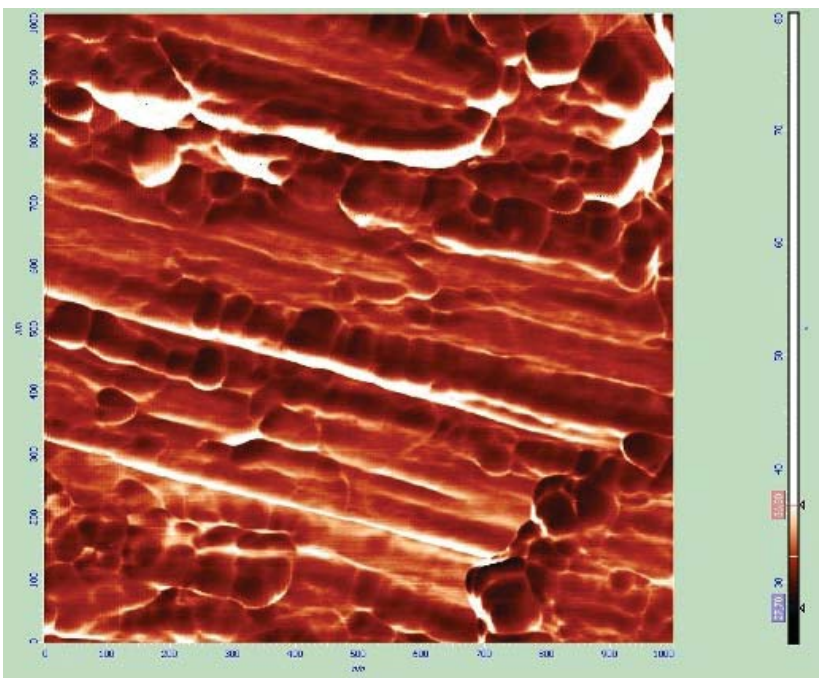

Рис. 1. Микроструктура поверхности шлифа с вакансионными кластерными трубками образца $\mathrm{Pb}-\mathrm{BKT}$, полученная методом АСМ

[Fig. 1. Microstructure of a surface of the thin section with vacancy cluster tubes of $\mathrm{Pb}$-VCT sample, obtained by the AFM method 


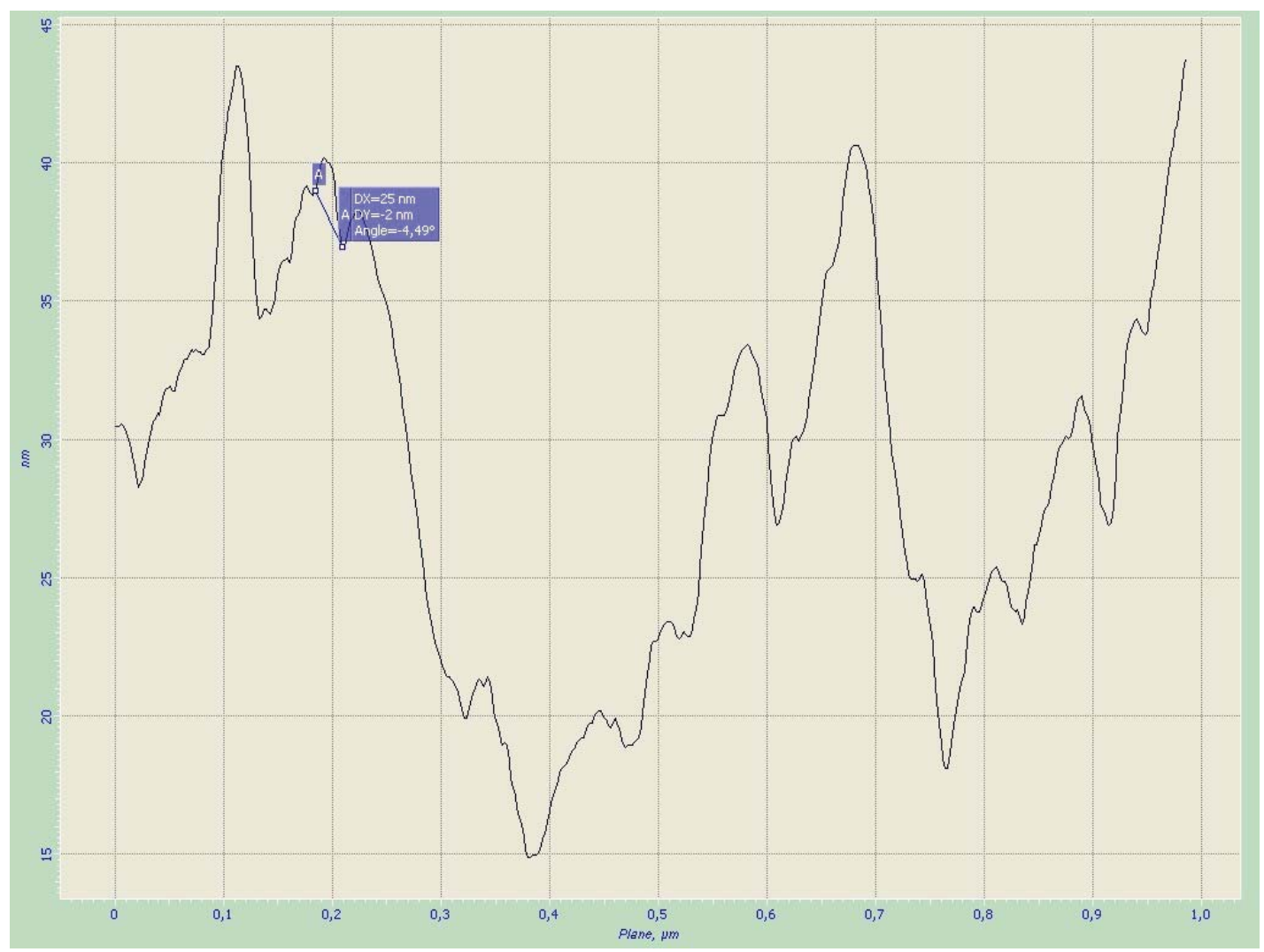

Рис. 2. Графическое изображение рельефа поверхностного образца Рb-ВКТ с вакансионными кластерными трубками. Характерные масштабы поверхности определялись с помощью спектра мощности (Фурье анализ) с применением программного обеспечении femtoscan onlin

[Fig. 2. Graphical representation of the relief of the surface sample Pb-VCT with vacancy cluster tubes. The characteristic surface scales were determined using a power spectrum (Fourier analysis) using the femtoscan onlin software]

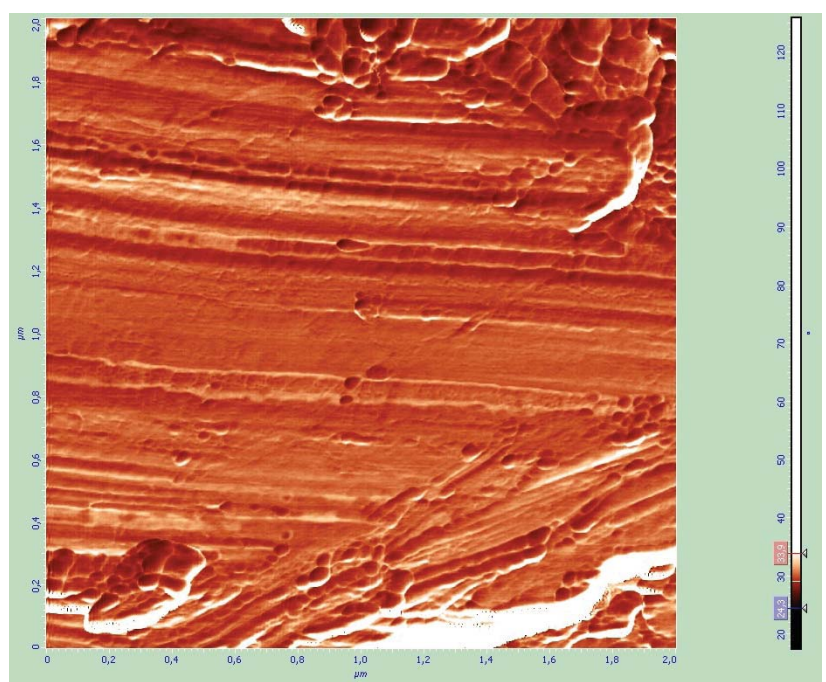

Рис. 3. Поверхности шлифа с вакансионными кластерными трубками образца Al-BКТ, полученная методом АCM

[Fig. 3. Surfaces of a section with vacancy cluster tubes of the Al-VCT sample, obtained by the AFM method] равновесных вакансий на уровне $10^{-3}-10^{-2}$. Столь высокая концентрация неравновесных вакансий, как указывалось выше, и приводит к их конденсации в вакансионные кластеры-трубки с гексагональной симметрией. С другой стороны, столь высокая стационарная концентрация неравновесных вакансий обуславливает возможность протекания процесса твердофазной перекристаллизации металла, следующего за первичной кристаллизацией. Процесс протекает в условиях, значительно удаленных от равновесных в сравнении с обычной кристаллизацией металла из расплава. Такие процессы могут приводить к образованию высокоупорядочных неравновесных состояний, характерных для неравновесных открытых систем (типа диссипативных структур).

Таким образом, можно предположить, что при высокоинтенсивной пластической деформации (ВИПД) на стадии кристаллизации расплава на фоне высокой стационарной концентрации неравновесных вакансий происходит образование нового типа эле- 

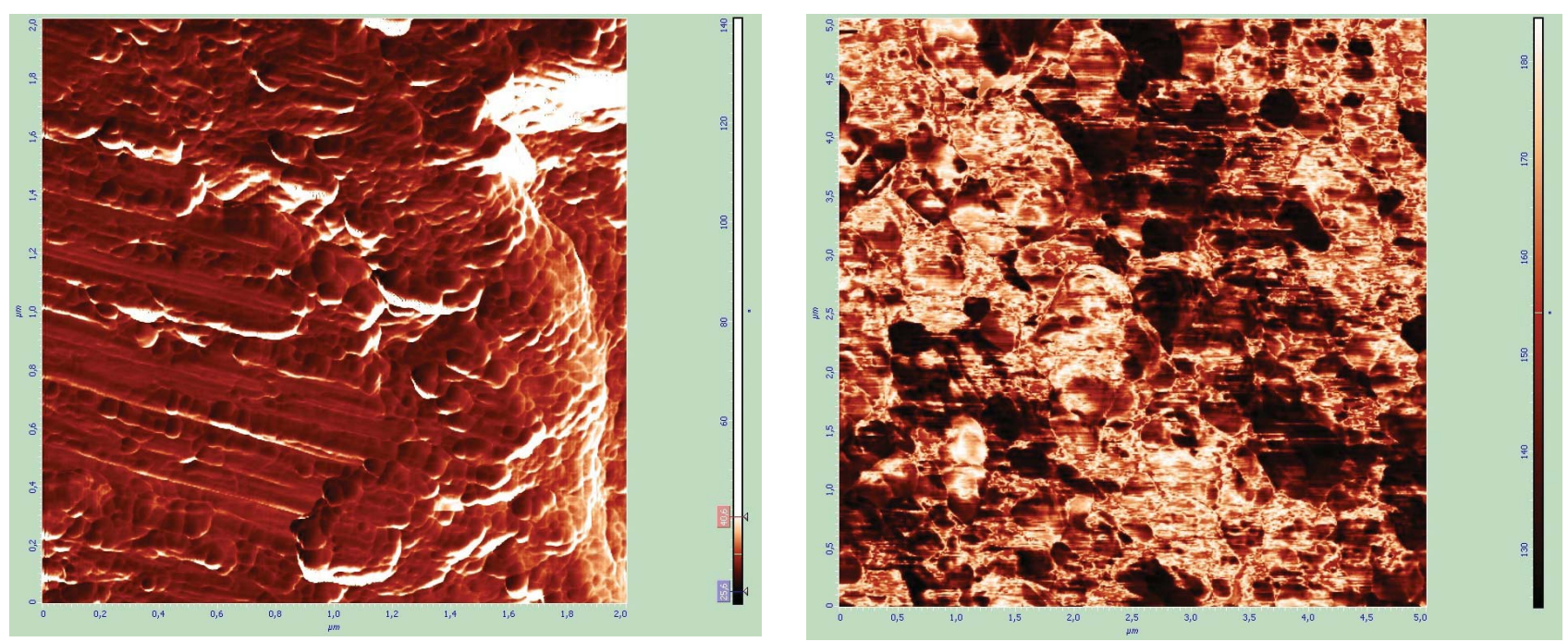

Рис. 4. Микроструктура поверхности шлифа с вакансионными кластерными трубками образца Al-BКТ, полученная методом АСМ: А) микроструктура с излома шлифа; В) микроструктура с торца шлифа

[Fig. 4. Microstructure of the surface of the section with vacancy cluster tubes of the Al-VCT sample obtained by the AFM method: A) microstructure from the fracture of the thin section; B) microstructure from the end of the section]

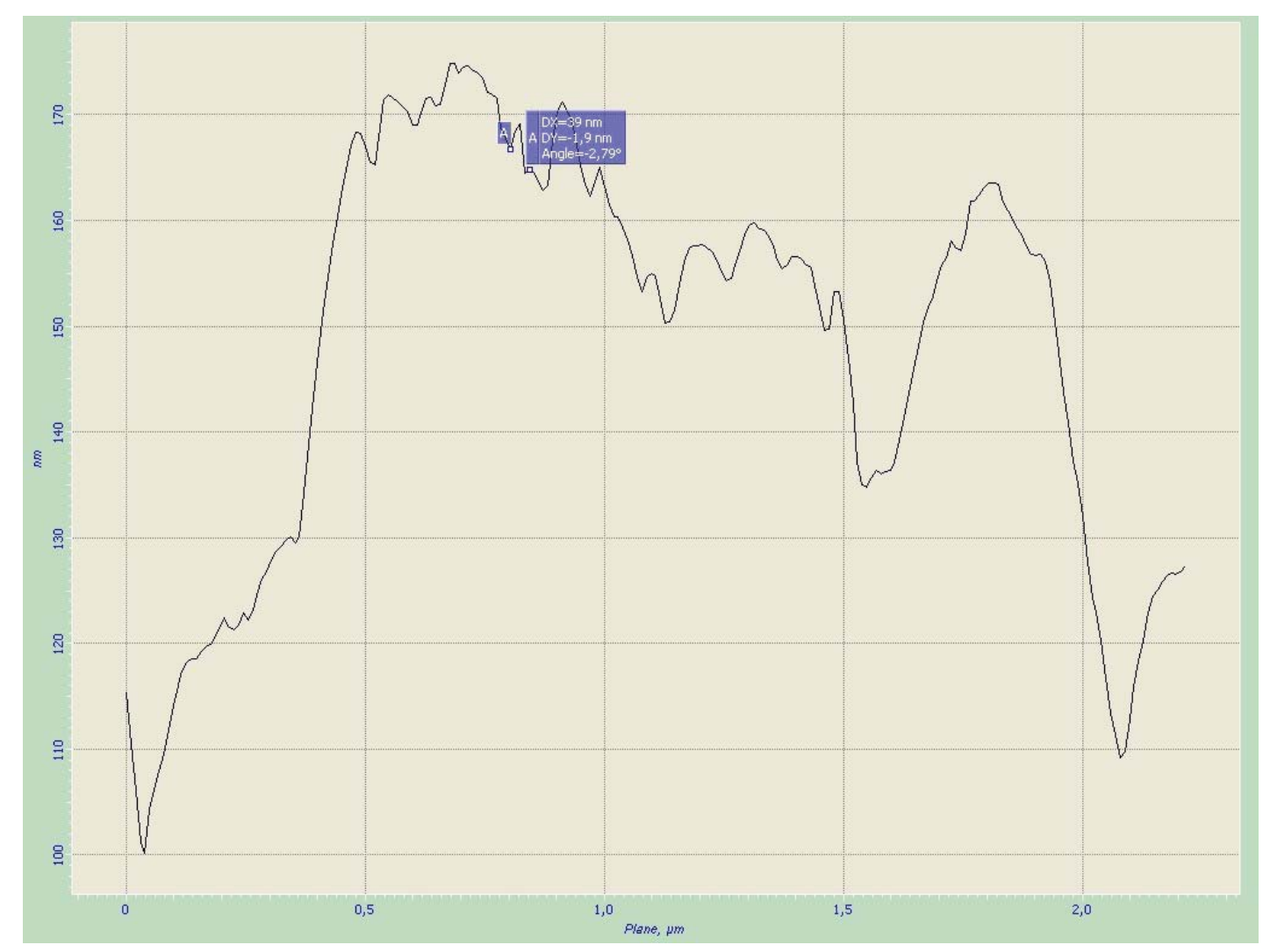

Рис. 5. Графическое изображение рельефа поверхностного образца Al-ВКТ с вакансионными кластерными трубками. Характерные масштабы поверхности определялись с помощью спектра мощности (Фурье анализ) с применением программного обеспечении femtoscan onlin

[Fig. 5. Graphical representation of the relief of the surface sample Al-VCT with vacancy cluster tubes. The characteristic surface scales were determined using a power spectrum (Fourier analysis) using the femtoscan onlin software] 
ментов структуры - вакансионных кластерных трубок (ВКТ). Физические процессы при этом можно интерпретировать следующим образом. Диссипация механической энергии при высокоинтенсивной сдвиговой деформации $[7,8]$ в условиях сверхскоростного (2000 об/мин) центробежного литья на аппаратах нового типа способствует образованию высокой концентрации неравновесных вакансий (порядка 10-2) в закристаллизованной части расплава, которые создают возможность для повторной перекристаллизации металла в твердой фазе (твердофазная перекристаллизация) в сильно неравновесных условиях с одновременной конденсацией неравновесных вакансий в поле центробежных сил в вакансионные кластеры в форме шестигранных трубок.

Образование в металлах субструктуры в виде структурных элементов нового типа - вакансионных кластерных трубок, могут, прежде всего, существенно повлиять на механические свойства металла [19-21]. На (рис. 6) представлено исследование динамических механических свойств образцов $\mathrm{Pb}$-исх. и $\mathrm{Pb}-\mathrm{BKT}$. На графиках представлены кривые, отображающие изменение модуля жесткости и модуля потерь исходного - $\mathrm{Pb}$-исх. и подвергну- того соответствующей обработке - $\mathrm{Pb}-\mathrm{BKT}$ образцов в процессе нагрева. Видно резкое увеличение модулей металла после обработки по сравнению с необработанным, и практически одинаковый ход кривых в процессе нагрева.

На рис. 7 представлены сравнительные исследования по изучению характера сверхпроводящего перехода при определении температуры сверхпроводящего перехода $(T)$ для образцов стандартного свинца (Pb «initial») с крупнокристаллической структурой и свинца (Pb «No. 1») после кристаллизации исходного стандартного свинца при центробежном литье со скоростью 2000 об/мин. Как следует из полученных результатов, характер перехода в сверхпроводящее состояние образца $\mathrm{Pb}$ «No. 1» существенно отличается от образца $\mathrm{Pb}$ «initial». Известно, что свинец является классическим примером низкотемпературного сверхпроводника первого рода. Переход в сверхпроводящее состояние образца $\mathrm{Pb}$ «No. 1» проходит по типу, свойственному сверхпроводникам второго рода. Причем температура $T_{c}$ для $\mathrm{Pb}$ «No. $1 »$ не совпадает с $T_{c}$ для $\mathrm{Pb}$ «initial» и несколько выше ( 0.025 $\mathrm{K})$, что может быть связано с наличием внутренних упругих напряжений в областях вакансионных клас-

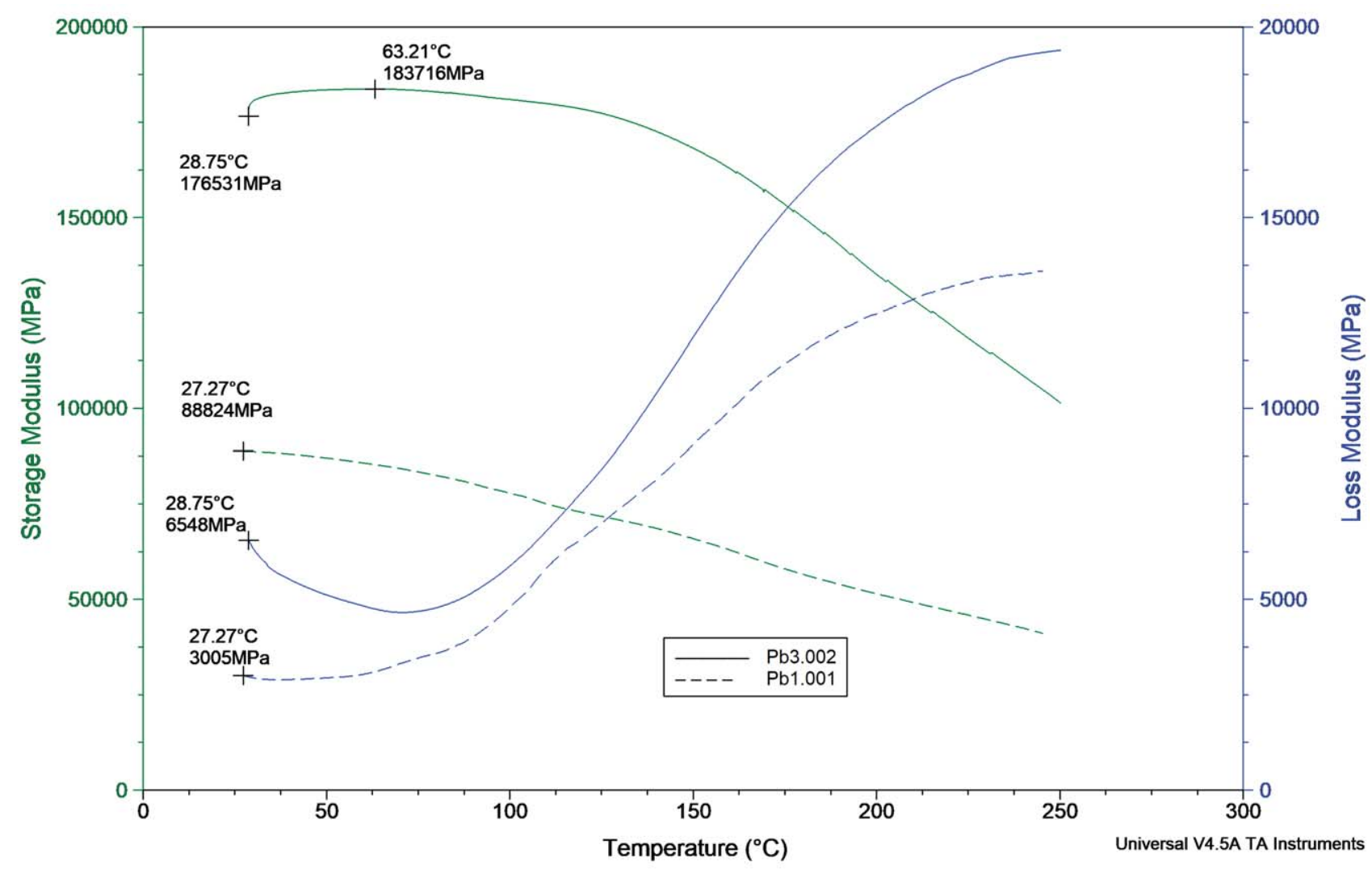

Рис. 6. Термограммы динамического механического анализа (ДМА) -----Pb1 исх. и ——Pb3-ВКТ

[Fig. 6. Dynamic mechanical analysis (DMA) thermograms ----- $\mathrm{Pb} 1$ initial and $\mathrm{Pb} 3-\mathrm{VCT}]$ 


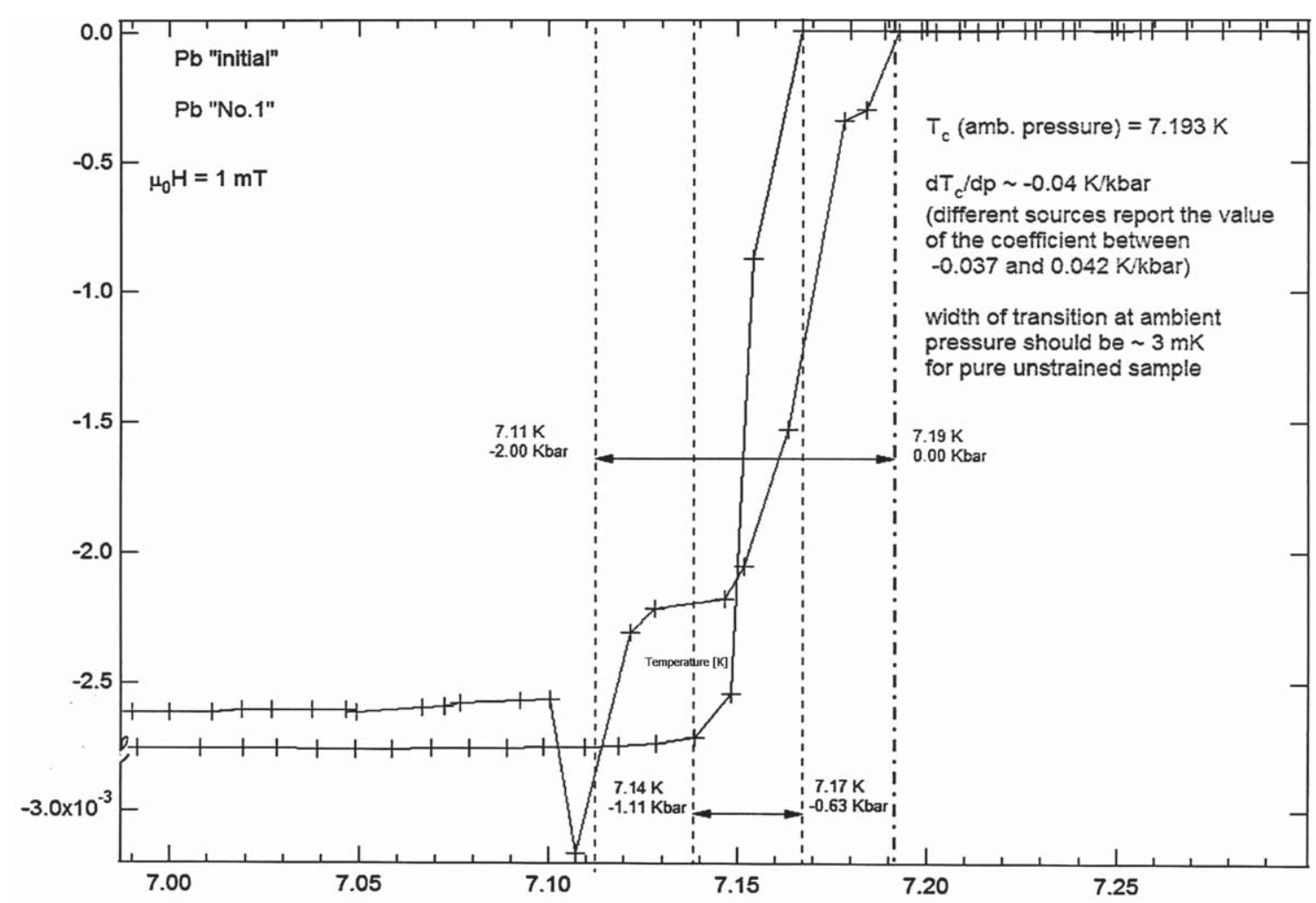

Рис. 7. Сравнительные исследования по изучению характера сверхпроводящего перехода при определении температуры сверхпроводящего перехода $\left(T_{c}\right)$ для образцов стандартного свинца $\mathrm{Pb}$ «initial» - 2 и для структурированного $\mathrm{Pb} \ll$ No.1»

[Fig. 7. Comparative studies of the nature of the superconducting transition during determining the superconducting transition temperature (Tc) for standard "initial" $\mathrm{Pb}-2$ and for Pb "No.1" with VCT-1]

терных трубок [9]. Такие напряжения могут приводить к растяжению кристаллической решетки в $\mathrm{Pb}$ «No. 1» и увеличению её параметра.

В заключение отметим, что на новых аппаратах в процессе центробежного литья при высокоинтенсивной пластической деформации $\left[\varepsilon^{\prime}=\left(10^{2}\right.\right.$ $\left.\left.10^{4}\right) \mathrm{c}^{-1}\right]$ на границе раздела «твердое-жидкое» создаются уникальные условия для получения металлов с собственной субструктурой вакансионных кластерных трубок (типа диссипативной структуры). Такая субструктра обуславливает существенное изменение механических и физико-химическими свойств, которые кардинально отличаются от традиционных свойств исходных металлов и фактически требуют сопоставительного изучения всех основных свойств подобным способом полученных металлов.

\section{ВЫВОДЫ}

1. При центробежном литье на аппаратах нового типа при скоростях вращения порядка 2000 об/мин был реализован процесс образования новой субстрктуры металлов $\mathrm{Al}$ и $\mathrm{Pb}$ в форме вакансионных кластерных трубок (ВКТ).
2. Физические процессы при этом можно интерпретировать следующим образом. Механическая энергия при сдвиговой деформации при центробежном литье на аппаратах нового типа идет на образование высокой концентрации неравновесных вакансий, которые создают возможность для кристаллизации металла в твердой фазе (твердофазная перекристаллизация) в сильно неравновесных условиях (при концентрации неравновесных вакансий в твердой фазе $10^{-2}$ ), с одновременным образованием структурированного материала в виде вакансионных кластерных трубок.

3. При центробежном литье на аппаратах нового типа при скоростях вращения порядка 2000 об/мин на фоне высокой концентрации неравновесных вакансий можно осуществлять твердофазный синтез тугоплавких соединений, например, таких как: $\mathrm{Al}_{4} \mathrm{C}_{3}, \mathrm{AlB}_{2}$ и т. п. и, соответственно, получать высокопрочные композиционные материалы на основе $\mathrm{Al}$ и других легкоплавких металлов, и не только легкоплавких, но и других конструкционных металлов.

4. Структурированные металлы и структурированные композиционные материалы с измененными 
механическими и физико-химическими свойствами могут механически обрабатываться и принимать любую конструкционную форму с новыми функциональными свойствами. Объемы производимых новых материалов могут достигать до нескольких сотен килограмм, а в перспективе и нескольких тонн!

Возможные практические приложения:

- Создание и разработка новых технологических направлений в области «атермической металлургии» с целью получения материалов с уникальными механическими и физико-химическими свойствами.

- Получение металлов с новыми функциональными свойствами.

- Кодирование систем защиты электронных систем передачи информации.

- Электронные замки для различных видов защиты.

- Защита ценных бумаг.

- Создание обособленных систем информационного обмена

- Создание суперакуумуляторов на основе $\mathrm{Pb}$, $\mathrm{Zn}-\mathrm{Ag}$.

\section{СПИСОК ЛИТЕРАТУРЫ}

1. Горелик С. С. Рекристаллизация металлов и сплавов. М.:, Металлургия, 1978, 486 с.

2. Лаповок В. Н., Новиков В. И., Свирида С. В. // $\Phi T T, 1983$, т. 25 , № 6, с. 1846-1848.

3. Новиков В. И., Трусов Л. И., Лаповок В. Н., Гелейшвили Т. П. // Порошковая металлургия, 1984, № 5, c. $28-34$.

4. Новиков В. И., Ганелин В. Я., Трусов Л. И. // ФТT, 1986, т. 28, № 4, с. 1251-1254.

5. Горелик С. С., Блантер М. С. // Изв. АН СССР. Металлы, 1982, № 2, с. 90-93.

6. Новиков В. И., Ганелин В. Я., Трусов Л. И. // Металлофизика, 1986, т. 8, № 2, с. 111-113.
7. Гегузин Я. Е., Парицкая Л. Н., Богданов В. В., Новиков В. И. // Физика металлов и металловедение, 1983 , т. 55, № 4, с. $768-773$.

8. Фридель Ж. Дислокации. / Пер. с англ. Ж. Ройтбурга, М.: Мир, 1967, 643 с.

9. Кан Р. Физическое металловедение. М.: Мир, 1968 , т. 3, 484 c.

10. Глейтер Г., Чалмерс Б. Большеугловые гранищы зерен. М.: Мир, 1975, 374 с.

11. Косевич В. М., Иевлев В. М., Палатник Л. С., Федоренко А. И. Структура межкристаллигных и межфазных грании. М.: Металлургия, 1980, 256 с.

12. Новиков. В. И., Грязнов В. Г., Трусов Л. И. // Физика, химия, механика, 1986, № 1, с. 134-139.

13. Новиков В. И., Лаповок В. Н., Свирида С. В. // Физика металлов и металловедение, 1984, т. 57, № 4, c. $718-721$.

14. Трусов Л. И., Новиков В. И., Грязнов В. Г. // Свойства и применение дисперсных порошков. Киев, Наукова думка, 1986, с. 98-114.

15. Новиков В. И., Трусов Л. И., Грязнов В. Г. Рост кристаллов. М.: Наука, 1988, т. 17, с. 69-86.

16. Новиков В. И., Свирида С. В., Трусов Л. И. // Металлофизика, 1984, т. 6, № 3, с. 114-115.

17. Утяшев Ф. З. // Физика и техника высоких давлений, 2010, т. 20, № 1, с. 7-25.

18. Бриджмен П. Исследование больших пластических деформаций и разрыва. М.: Издательство Иностр. лит., 1955.

19. Трусов Л. И., Новиков В. И., Репин И. А., Казилин Е. Е., Ганелин В. Я. // Металлофизика, 1988, т. 10, № 1, c. 104-107.

20. Trusov L. I., Khvostantseva T. P., Solov'ev V. A., Mel'nikova V. A. // J. of Materials Science, 1995, vol. 30, № 11, pp. 2956-2961. DOI: https://doi.org/10.1007/ BF00349669

21. Trusov L. I., Khvostantseva T. P., Solov'ev V. A., Mel'nikova V. A. // Nanostructured Materials, 1994, vol. 4, № 7, pp. 803-813. DOI: https://doi.org/10.1016/09659773(94)90086-8

\title{
PECULIARITIES OF STRUCTURING FOR MASS CRYSTALLIZATION OF Al AND Pb MELTS IN CONDITIONS OF HIGH-INTENSITY PLASTIC DEFORMATION AT CENTRIFUGING
}

\author{
(C) 2018 Ju. I. Tarasov, V. V. Krjachko, V. I. Novikov \\ OOO «SVETOZAR», 1/2 Soljanka, 109022 Moscow, Russia \\ e-mail:vinovikov@redstaratom.ru
}

Received 14.02.2018

\begin{abstract}
Presents experimental results of $\mathrm{Al}$ and $\mathrm{Pb}$ metals crystallization carried out under highintensity plastic deformation (HIPD) $\left[\varepsilon^{\prime}=\left(10^{2}-10^{4}\right) \mathrm{sec}^{-1}\right]$ reaching the level of so called "solid-liquid" state in the new type of centrifugal casting device at rotor speeds of up to $2000 \mathrm{rpm}$. Using the
\end{abstract}


method of atomic force microscopy (AFM), vacancy cluster tubes (VCT) with average diameters of $39 \mathrm{~nm}$ for $\mathrm{Al}$ and $25 \mathrm{~nm}$ for $\mathrm{Pb}$ have been detected in the crystallized volume of $\mathrm{Al}$ and $\mathrm{Pb}$ metals. Physical model of the formation of a new substructure within the metals in the form of vacancy cluster tubes, received in the process of high-intensive plastic deformation (HIPD) during the process of mass crystallization of $\mathrm{Al}$ and $\mathrm{Pb}$ melts, and, also the changes in the mechanical, magnetic and superconducting properties of the above metals, which followed this process.

When crystallizing $\mathrm{Al}$ and $\mathrm{Pb}$ under high-intensive plastic deformation (HIPD) of $\varepsilon^{\prime}=\left(10^{2}-10^{4}\right)$ per second type, in high-speed centrifugal casting devices, specially selected modes of metal crystallization are being chosen and special conditions are being created to achieve the dimensional effect of dynamic (shift) re-crystallization [1]. Shift deformation during centrifugal crystallization is caused primarily by a large incline of the temperature field from the periphery (relative to the cold wall of the rotor) to the molten central part of the rotor. The difference in the angular movement velocities of the already-frozen part of the metal (adjacent to the outer surface of the rotor wall) and the central part, where the metal still remains in the molten state, leads to a high-intensity deformation $\left[\varepsilon^{\prime}=\left(10^{2}\right.\right.$ $10^{4}$ ) per $\mathrm{sec}^{-1}$ ] of the crystallized metal melt solidified phase. Since the grain sizes at the crystallized phase initially comprise around tens of nano-meters (approximately crystal nucleation size), it becomes possible to achieve the dimensional effect of the dynamic re-crystallization [1] of a "nanocrystalline" solidified metal at high shift of strain velocities. The "non-equilibrium vacancies" formed this way condense into vacancy clusters, which are formed in the centrifugal force field in the form of vacancy-shaped cluster tubes stretched out to the center of rotation of the rotor.

The process undergoes conditions that are considerably different from the "equilibrium" conditions as compared to the ordinary metal crystallization from the melt. Such processes can lead to the formation of highly ordered non-equilibrium states characteristic of non-equilibrium open systems.

Keywords: intensive plastic deformation, dynamic recrystallization, nonequilibrium vacancies, vacancy cluster tubes, solid phase recrystallization.

DOI: https://doi.org/10.17308/kcmf.2018.20/484

\section{REFERENCES}

1. Gorelik S. S. Rekristallizacija metallov i splavov [Recrystallization of Metals and Alloys]. Moscow, Metallurgija Publ., 1978, p. 486. (in Russ.)

2. Lapovok V. N., Novikov V. I., Svirida S. V. Fizika tverdogo tela, 1983, vol. 25, no. 6, pp. 1846-1848. (in Russ.)

3. Novikov V. I., Trusov L. I., Lapovok V. N., Gelejshvili T. P. Poroshkovaja metallurgija [Powder metallurgy], 1984, no. 5, pp. 28-34. (in Russ.)

4. Novikov V. I., Ganelin V. Ja., Trusov L. I., Fizika tverdogo tela, 1986, vol. 28, no. 4, pp. 1251-1254. (in Russ.)

5. Gorelik S. S., Blanter M. S. Izv. AN SSSR. Metally [Metals], 1982, no. 2, pp. 90-93. (in Russ.)

6. Novikov V. I., Ganelin V. Ja., Trusov L. I. Metallofizika [Metal Physics], 1986, vol. 8, no. 2, pp. 111-113. (in Russ.)

7. Geguzin Ja. E., Parickaja L. N., Bogdanov V. V., Novikov V. I. Fizika Metallov i Metallovedenie [Physics of Metals and Metallography], 1983, vol. 55, no. 4, pp. 768773. (in Russ.)

8. Friedel J. Dislocations. Oxford, Pergamon Press, 1964, 587 p.

9. Physical Metallurgy. / Ed. by R.W. Cahn, P. Haasen, Amsterdam, New York, North-Holland Physics Pub., 1996, 2888 p.
10. Gleiter H., Chalmers B. High-angle Grain Boundaries. Oxford, Pergamon Press, 1972. 303 p.

11. Kosevich V. M., Ievlev V. M., Palatnik L. S., Fedorenko A. I. Struktura mezhkristallignyh i mezhfaznyh granic [Structure of Intercrystalline and Interphase Boundaries], Moscow, Metallurgija Publ., 1980, p. 256. (in Russ.)

12. Novikov. V. I., Grjaznov V. G., Trusov L. I. Fizika, himija, mehanika [Physics, Chemistry, Mechanics], 1986, no. 1, pp. 134-139. (in Russ.)

13. Novikov V. I., Lapovok V. N., Svirida S. V. Fizika Metallov i Metallovedenie [Physics of Metals and Metallography], 1984, vol. 57, no. 4, pp. 718-721. (in Russ.)

14. Trusov L. I., Novikov V. I., Gryaznov V. G. Svojstva i primenenie dispersnyh poroshkov [Properties and Application of Dispersed Powders], Kiev, Naukova Dumka Publ., 1986, pp. 98-114. (in Russ.)

15. Novikov V. I., Trusov L. I., Grjaznov V. G. Rost kristallov [Growth of Crystals], Moscow, Nauka Publ., 1988, vol. 17, pp. 69-86. (in Russ.)

16. Novikov V. I., Svirida S. V., Trusov L. I. Metallofizika [Metal Physics], 1984, vol. 6, no. 3, pp. 114-115. (in Russ.)

17. Utyashev F. Z. Fizika i tehnika vysokih davlenij [High-pressure Physics and Technology], 2010, vol. 20, no. 1, pp. 7-25. (in Russ.)

18. Bridgman P. W. Studies in Large Plastic Flow and Fracture. McGraw Hill, 1952, 362 p. 
19. Trusov L. I., Novikov V. I., Repin I. A., Kazilin E. E., Ganelin V. Ja. Metallofizika [Metal Physics], 1988, vol. 10, no.1, pp. 104-107. (in Russ.)

20. Trusov L. I., Khvostantseva T. P., Solov'ev V. A., Mel'nikova V. A. Journal of Materials Science, 1995, vol.
30, no. 11, pp. 2956-2961. DOI: https://doi.org/10.1007/ BF00349669

21. Trusov L. I., Khvostantseva T. P., Solov'ev V. A., Mel'nikova V. A. Nanostructured Materials, 1994, vol. 4, no. 7, pp. 803-813. DOI: https://doi.org/10.1016/09659773(94)90086-8
Тарасов Юрий Иванович - д. экон. н., президент компании ООО «СВЕТОЗАР»; тел.: +7(916) 4950698, e-mail: y.tarasov@list.ru

Крячко Валерий Викторович - к. геол.-минерал. н., ведущий научный сотрудник ООО «СВЕТОЗАР», тел.: +7(980) 5477531, e-mail: platdep@mail.ru

Новиков Виктор Иванович - к. т. н., технический директор ООО «СВЕТОЗАР»; тел.: +7(915) 0619836, e-mail: novikov-nanotech@yan
Yuriy I. Tarasov - Dr. Sci. (Econ.), the President of the company LLC "SVETOZAR"; tel.: +7(916) 4950698, e-mail: y.tarasov@list.ru

Valery V. Kryachko - Cand. Sci. (Geol. and Mineral.), Leading Researcher of the company LLC "SVETOZAR"; tel.:+7 (980) 5477531, e-mail: platdep@mail.ru

Victor I. Novikov - Cand. Sci. (Eng.), Technical Director of LLC "SVETOZAR"; tel.: +7(915) 0619836, e-mail: novikov-nanotech@yan 